\title{
An up-down determination of the required seated duration after intrathecal injection of bupivacaine and fentanyl for the prevention of hypotension during Cesarean delivery Détermination, à l'aide d'une méthode en escalier, de la durée requise en position assise après une injection intrathécale de bupivacaïne et de fentanyl pour prévenir l'hypotension pendant un accouchement par césarienne
}

\author{
Albert Moore, MD · Eva el-Mouallem, MD • Aly el-Bahrawy, MD • Ian Kaufman, MD • \\ Mahmoud Moustafa, MD • Simone Derzi, MD • Roupen Hatzakorzian, MD • \\ William Lipishan, MD
}

Received: 24 January 2017/Revised: 3 May 2017/Accepted: 5 July 2017 / Published online: 24 July 2017

(C) Canadian Anesthesiologists' Society 2017

\begin{abstract}
Purpose A prolonged seated time after intrathecal injection of hyperbaric bupivacaine and morphine is related to the incidence of hypotension during Cesarean delivery, but results in a high incidence of pain during peritoneal closure. We conducted this study to determine the effect of the addition of intrathecal fentanyl on the relationship between seated time and hypotension and intraoperative analgesia requirements.

Methods Women undergoing Cesarean delivery were randomized to receive an intrathecal injection of either 11.25 or $15 \mathrm{mg}$ of hyperbaric bupivacaine with morphine $150 \mu \mathrm{g}$ and fentanyl $15 \mu \mathrm{g}$ using a combined spinalepidural technique. The seated duration following intrathecal injection was assigned using up-down methodology. If the preceding patient was hypo- or normotensive, the next patient sat for 15 sec more or less, respectively. A systolic blood pressure $<80 \%$ of the preoperative value was defined as hypotension; a standardized anesthetic was administered, and the
\end{abstract}

This article is accompanied by an editorial. Please see Can J Anesth 2017; 64: this issue.

A. Moore, MD $(\bowtie) \cdot$ E. el-Mouallem, MD · A. el-Bahrawy, MD

I. Kaufman, MD · M. Moustafa, MD · S. Derzi, MD ·

R. Hatzakorzian, MD · W. Lipishan, MD

Department of Anesthesia, Royal Victoria Hospital, McGill

University Health Centre, 1001 Decarie Boulevard, Montreal, QC,

Canada

e-mail: moore_albert@hotmail.com presence of pain during the procedure was recorded. Isotonic regression of pooled adjacent violators was used to determine the time at which $50 \%$ of each group would avoid hypotension (i.e., the median effective seated time). Results There were 15 patients in each group. The median seated time was $129 \mathrm{sec}$ (95\% confidence interval [CI], 116 to 150) for the 11.25-mg group and $459 \mathrm{sec}$ (95\% CI, 444 to 471) for the 15-mg group. Only one (3\%) of the 30 patients in the study had pain on peritoneal closure, and this was successfully treated with intravenous fentanyl.

Conclusion We have determined the seated time required following intrathecal injection of hyperbaric bupivacaine, morphine, and fentanyl to prevent hypotension in $50 \%$ of patients undergoing Cesarean delivery.

Trial registration www.clinicaltrials.gov, NCT01896960. Registered 2 July 2013.

\section{Résumé}

Objectif La prolongation du temps passé en position assise après une injection intrathécale de bupivacaïne hyperbare et de morphine est liée à l'incidence d'hypotension pendant un accouchement par césarienne, mais cette approche entraîne une forte incidence de douleur pendant la fermeture du péritoine. Nous avons réalisé cette étude afin de déterminer l'effet de l'ajout de fentanyl intrathécal sur la relation entre le temps passé en position assise, l'hypotension et les besoins analgésiques peropératoires. 
Méthode Des femmes subissant un accouchement par césarienne ont été randomisées à recevoir une injection intrathécale de 11,25 ou $15 \mathrm{mg}$ de bupivacaïne hyperbare avec $150 \mu \mathrm{g}$ de morphine et $15 \mu \mathrm{g}$ de fentanyl via une technique de rachi-péridurale combinée. La durée en position assise après l'injection intrathécale a été attribuée à l'aide d'une méthodologie de l'escalier. Si la patiente précédente était hypo- ou normotendue, la patiente suivante restait assise pendant $15 \mathrm{sec}$ de plus ou de moins, respectivement. Une tension artérielle systolique < $80 \%$ de la valeur préopératoire était définie en tant qu'hypotension; l'anesthésie était standardisée, et la présence de douleur pendant l'intervention a été enregistrée. Une régression isotonique de l'algorithme PAV (pour pooled-adjacent-violators) a été utilisée pour déterminer le moment auquel $50 \%$ de chaque groupe éviterait l'hypotension (c.-à-d. le temps efficace médian en position assise).

Résultats $A u$ total, chaque groupe était composé de 15 patientes. Le temps médian en position assise était de 129 sec (intervalle de confiance [IC] $95 \%, 116$ à 150) pour le groupe 11,25 mg et de $459 \mathrm{sec}$ (IC $95 \%, 444$ à 471) pour le groupe $15 \mathrm{mg}$. Seule une (3\%) des 30 patientes à l'étude a ressenti de la douleur lors de la fermeture du péritoine, laquelle a été traitée avec succès à l'aide de fentanyl intraveineux.

Conclusion Nous avons pu déterminer le temps en position assise nécessaire suite à une injection intrathécale de bupivacaïne hyperbare, de morphine et de fentanyl pour prévenir l'hypotension chez $50 \%$ des patientes subissant un accouchement par césarienne.

Enregistrement de l'étude www.clinicaltrials.gov, NCT01896960. Enregistrée le 2 juillet 2013.

Hypotension is common after spinal anesthesia for Cesarean delivery. ${ }^{1}$ Many techniques, often in combination, are utilized to aid in its prevention. These include a reduction in the dose of local anesthetic, ${ }^{2}$ uterine displacement, ${ }^{3}$ fluid loading, ${ }^{4}$ and prophylactic vasopressor infusions. ${ }^{5}$ Another investigational technique is to prolong the seated time after intrathecal injection. This technique may limit upward spread of hyperbaric local anesthesia and the resultant hypotension. Nevertheless, the limited studies of this technique show conflicting results. Kohler et al. found that women undergoing Cesarean delivery who sat for three minutes after an intrathecal injection of hyperbaric bupivacaine $14 \mathrm{mg}$ did not have lower hypotension rates or reduced vasopressor requirements. ${ }^{6}$ El-Hakeem et al. found that leaving patients seated for five minutes after an intrathecal injection of hyperbaric bupivacaine $10 \mathrm{mg}$ did reduce vasopressor requirements. ${ }^{7}$ The reason for these differing results may have been related to the choice of seated times and bupivacaine dose. Since the actual relationship between seated duration and hypotension has not been established, the appropriate "dose" of seated time for the amount of bupivacaine administered may not have been studied. In an initial attempt to characterize the dose response relationship between seated time and hypotension, we ascertained that a seated time existed where $50 \%$ of parturients avoided hypotension (i.e., median seated time) and that it differed for bupivacaine dose. In patients who received spinal anesthesia consisting of morphine and either $11.25 \mathrm{mg}$ or $15 \mathrm{mg}$ of hyperbaric bupivacaine, the median seated time was $130 \mathrm{sec}(95 \%$ confidence interval [CI], 117 to 150$)$ and $385 \mathrm{sec}$ (95\% CI, 381 to 396), respectively. ${ }^{8}$ Nevertheless, in this study, $22 \%$ of the patients required supplemental analgesia for peritoneal closure. Similar to low-dose spinal techniques in this population, ${ }^{2}$ extended seated times attempt to reduce sympathetic blockade by reducing the spread of local anesthesia, which may result in unacceptable levels of supplemental analgesia requirements. Nevertheless, El-Hakeem found no difference in the supplemental analgesia requirements between patients left seated for five minutes and those placed immediately supine. This result may have been due to the inclusion of fentanyl in the intrathecal anesthetic. The addition of a lipophilic opioid can decrease supplemental analgesia requirements, ${ }^{9}$ but it may also affect spinal blockade characteristics ${ }^{10}$ and result in earlier requirements for vasopressors. ${ }^{11}$ It is unknown how the changes in spinal blockade induced by intrathecal fentanyl might affect the relationship between seated time and the incidence of hypotension. It is also unknown if an extended seated time will alter the analgesic effects of intrathecal fentanyl.

We designed this study to determine the median seated time for the prevention of hypotension after intrathecal injection of either $11.25 \mathrm{mg}$ or $15 \mathrm{mg}$ of hyperbaric bupivacaine with preservative-free morphine and fentanyl. Our secondary objective was to measure the rates of supplemental analgesia requirements for patients with extended seated duration.

\section{Methods}

Approval for this study was obtained from the McGill University Health Centre Research Ethics Office, and all participants provided written informed consent.

We included women aged 18-45 yr undergoing scheduled Cesarean delivery. We excluded women with 
hypertensive disease, multiple gestations, fetal or placental abnormalities, American Society of Anesthesiologists classification $>$ II, a body mass index $>35$, an inability to communicate in English or French, or those with contraindications to neuraxial anesthesia or study medications.

A computer-generated randomization plan assigning patients to $11.25 \mathrm{mg}$ or $15 \mathrm{mg}$ of $0.75 \%$ hyperbaric bupivacaine was placed in sequentially numbered sealed envelopes that were opened after epidural placement. A blinded observer who was not present during the placement of neuraxial anesthesia assessed the patients' blood pressure and determined when hypotension was present.

In the preoperative holding area, baseline blood pressure was determined by taking the average of three noninvasive blood pressure measurements with the patient in the semirecumbent position. In the operating room, standard noninvasive monitors were placed. A two-puncture combined spinal-epidural anesthesia technique was utilized. With the patient in the seated position, a $16 \mathrm{G}$ Tuohy needle was introduced into the L3-4 vertebral interspace (confirmed by ultrasound). We identified the epidural space by loss of resistance with $<3 \mathrm{~mL}$ of saline and then placed a multi-orifice 20G epidural catheter (B. Braun Medical, Inc, Bethlehem, PA, USA). No test dose was given through the catheter. Following negative aspiration and catheter fixation, a $27 \mathrm{G}$ Whitacre needle was introduced into the L4-5 vertebral interspace (confirmed by ultrasound) and advanced until cerebrospinal fluid was obtained. The spinal medication (1.5 or $2.0 \mathrm{~mL}$ of $0.75 \%$ hyperbaric bupivacaine with preservative-free morphine $150 \mu \mathrm{g}$ and fentanyl $15 \mu \mathrm{g}$ ) was injected over $30 \mathrm{sec}$. After the injection, a noninvasive blood pressure cuff was set to measure blood pressure every minute. Patients remained seated for the predetermined time period (see below) and were then placed in the supine position with a wedge $(10 \times 30 \times 60$ $\mathrm{cm})$ under their right hip. A single fetal heart rate was obtained by Doppler. No fluid preload was provided to the patient. A litre bag of normal saline connected to an $18 \mathrm{G}$ intravenous cannula was hung 4-5 feet above the centre of the table and left wide open until a $10 \mathrm{~mL} \mathrm{~kg}^{-1}$ coload was administered. The intravenous was then slowed to a "keep vein open" rate. Surgical preparations were continued, and incision was allowed after a T6 blockade was confirmed with ice testing. We continued to measure the blood pressure every minute. The patient was considered hypotensive if a pre-delivery systolic blood pressure measurement was $<80 \%$ of baseline, or if symptoms of nausea, vomiting, or faintness were displayed. At this point, or after delivery, blood pressure management was then left to the discretion of the treating anesthesiologist. If the patient reported any abdominal pain $>0 / 10$ during the procedure, they were given a fentanyl $50 \mu \mathrm{g} i v$ bolus and, if this was not effective, an additional $5 \mathrm{~mL} i v$ bolus of $2 \%$ lidocaine.

We collected the patients' baseline demographic information. Once each patient was placed supine, a blinded observer (who was not present while the patient was seated) entered the room and recorded the following information: surgical duration, use of supplemental analgesia, and blood pressure readings until delivery. They also stated when the blood pressure fell to $<80 \%$ of the baseline systolic pressure. The observer measured the thoracic level of the dermatome blockade by moving ice cranially until the patient reported feeling cold. For the first blockade measurement, the starting point for the ice was the L5 dermatome, and for subsequent measurements, the starting point was two dermatome levels below the blockade determined in the previous measurement.

We based the seated duration on results from a previous study, ${ }^{8}$ and therefore, the initial patient in the $11.25-\mathrm{mg}$ and $15-\mathrm{mg}$ groups sat for $150 \mathrm{sec}$ and $390 \mathrm{sec}$, respectively. Following up-down methodology, the seated time for any subsequent patient depended on the hypotensive response of the previous patient. If the previous patient in the same group was hypotensive, the subsequent patient sat for 15 sec longer, and if the previous patient was not hypotensive, the subsequent patient sat for $15 \mathrm{sec}$ less. If a patient did not develop a T6 blockade within $15 \mathrm{~min}$ of being placed supine, the epidural could be utilized to provide anesthesia at the discretion of the treating anesthesiologist. The subsequent patient in the same bupivacaine group was then assigned the same seated duration.

In up-down methodology, a reliable estimate of a median dose can be determined after six changes from one state to another. ${ }^{12}$ We initially randomized 20 patients in each group and planned to stop enrolment after six so-called "crossovers" occurred. The first crossover in the 15-mg group was followed by a run of four patients who did not change status and then by a change in status every two or three patients. As the estimates of effective doses are more reliable when examined doses are close to the effective point, ${ }^{13}$ we decided to continue enrolment until we had six crossovers that clustered around a seated time. We included all the crossovers in the analysis and continued enrolling in both groups to maintain randomization.

Continuous variables are presented as mean (standard deviation) and compared using Student's $t$ tests. Ordinal variables are presented as median [interquartile range (IQR)] and compared with Wilcoxon rank-sum tests. Proportions are presented as numbers (\%) and are compared with Fisher's exact tests. We performed isotonic regression using the pooled-adjacent-violators algorithm (PAVA) by Pace and Stylianou and we used linear interpolation to calculate the third estimator with 
bootstrapped confidence intervals for the median and $95 \%$ effective seated time. ${ }^{13-15}$ We used the Stata/SE ${ }^{\mathrm{TM}}$ package version 14 (StataCorp LP, College Station, TX, USA) for statistical analysis and the R statistical package version 3.0.2 (R Foundation for Statistical Computing, Vienna, Austria) for isotonic regression.

\section{Results}

Figure 1 represents the flow of patients through the study. From July 2013 to October 2016, we assessed 90 patients, approached 82, and enrolled 30. The Table summarizes the baseline and operative characteristics of both groups. One patient in the 11.25-mg group did not develop a blockade following spinal anesthesia. Ice testing up to $15 \mathrm{~min}$ after the patient was placed supine showed no sensory blockade; consequently, the anesthesiologist used the epidural catheter successfully for surgical anesthesia. This patient was not included in determining the effective seated times, and the following patient was assigned the same seated duration. All other patients developed a T6 blockade and showed no lower limb movement during surgery. No patient developed hypotension while seated or fetal heart rate abnormalities before incision. The median seated time was $129 \mathrm{sec}(95 \% \mathrm{CI}, 116$ to 150$)$ for the $11.25-\mathrm{mg}$ group and $459 \mathrm{sec}$ (95\% CI, 444 to 471$)$ for the 15 -mg group. The 95\% effective seated time was estimated to be $162 \mathrm{sec}$ (95\% CI, 148 to 164) for the $11.25-\mathrm{mg}$ group and $477 \mathrm{sec}$ (95\% CI, 463 to 479 ) for the 15-mg group. Only one (3\%) of the 30 patients studied had pain on peritoneal closure, which was successfully treated with intravenous fentanyl (Fig. 2). Figure 3 characterizes the development of dermatome blockade over time, as determined by ice testing for each group. Figure 4 presents the PAVA and observed probabilities for each seated time studied.

\section{Discussion}

Leaving a patient seated for a prolonged period of time after intrathecal injection of hyperbaric local anesthetic is a simple technique that could theoretically decrease the spread of hyperbaric local anesthetic and the resulting hypotension during Cesarean delivery. The most obvious unintended consequence, however, would be inadequate anesthesia. The downward diffusion of hyperbaric local anesthesia could result in a blockade that is not high enough, or doesn't last long enough, for Cesarean delivery. This is a complication associated with low-dose spinal anesthesia. ${ }^{2}$ Nevertheless, compared with studies on lowdose spinal anesthesia, there are very few controlled trials comparing extended seated times with standard therapies for hypotension prevention, and the relative advantages and

Table Baseline characteristics of the parturients randomized to receive an intrathecal injection of 11.25 or $15 \mathrm{mg}$ of hyperbaric bupivacaine for Cesarean delivery

\begin{tabular}{lcc}
\hline & $\begin{array}{l}11.25 \mathrm{mg} \\
n=14\end{array}$ & $\begin{array}{c}15 \mathrm{mg} \\
n=15\end{array}$ \\
\hline Age (yr) & $33(3)$ & $34(4)$ \\
Height (cm) & $164(6)$ & $164(7)$ \\
Weight (kg) & $76.4(6.6)$ & $77.1(11.5)$ \\
Nulliparous & $13(87 \%)$ & $9(60 \%)$ \\
Gestational age (weeks) & $39.1(0.6)$ & $39.0(0.7)$ \\
Neonatal weight (g) & $3,259(92)$ & $3,334(152)$ \\
Time from placed supine until incision & $8.8(2.4)$ & $8.2(2.1)$ \\
$\quad$ (min) & $10.2(6.5)$ & $9.2(5.7)$ \\
Time from incision until delivery (min) & $37.9(10.5)$ & $33.1(13.0)$ \\
Time from delivery until skin closure & & \\
$\quad$ (min) & & \\
\hline
\end{tabular}

Results are presented as mean (standard deviation) or number (percent)

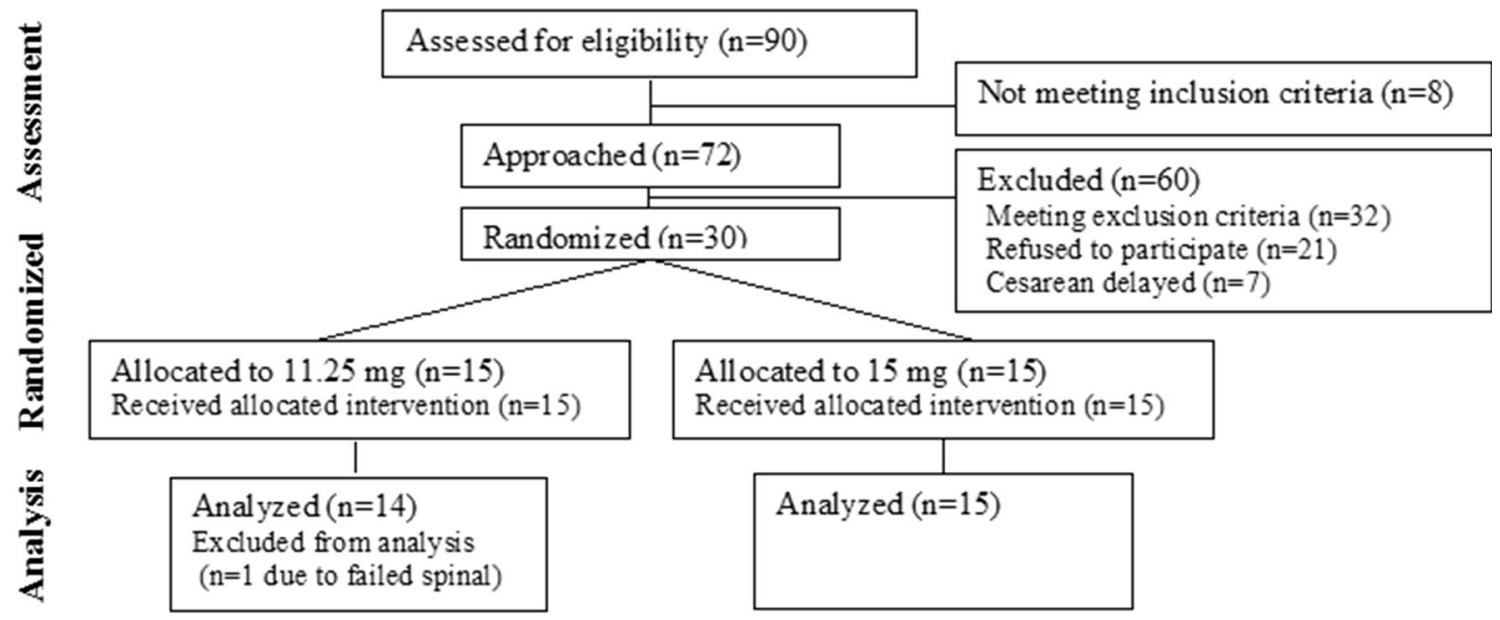

Fig. 1 The patient flow through the study 
Fig. 2 The seated times after intrathecal injection of 11.25 or $15 \mathrm{mg}$ of hyperbaric bupivacaine, fentanyl, and morphine and the resultant hypotensive response for patients undergoing Cesarean delivery

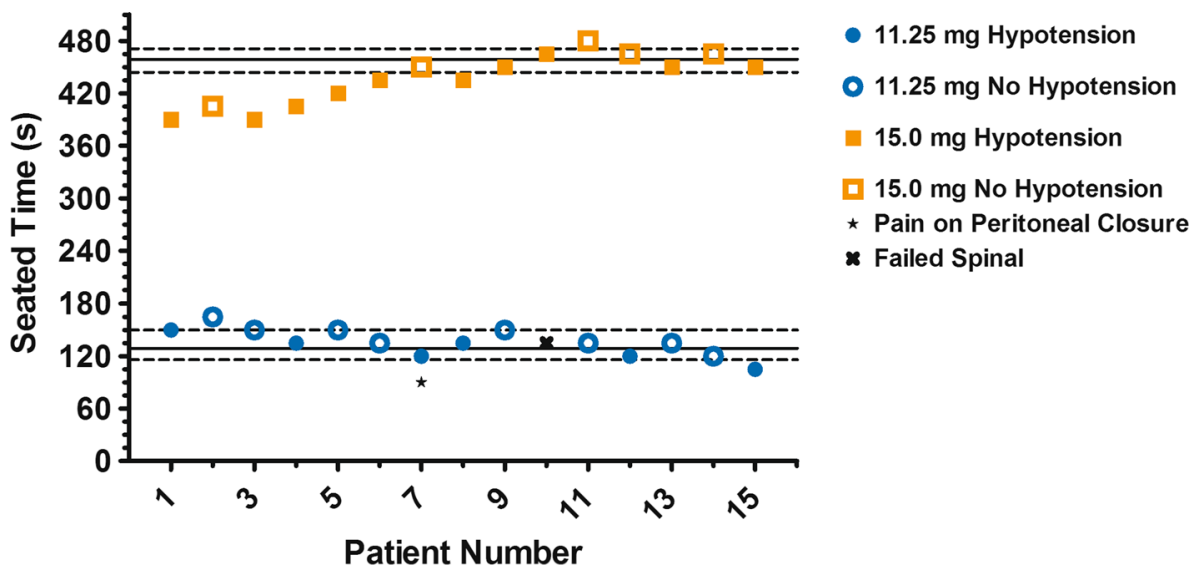

disadvantages of this technique are unproven. The appropriate levels of treatment should be determined prior to rigorous comparison of hypotension prevention techniques. The dose-response relationship of seated time with hypotension during Cesarean delivery is incompletely elucidated.

Using up-down methodology, we have determined the median point on the dose-response curve for seated time

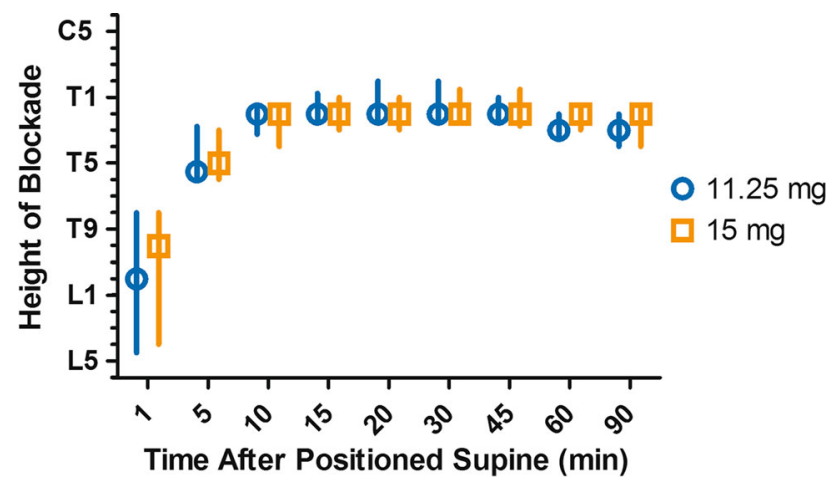

Fig. 3 The median [interquartile range] cranial dermatome blockade as measured by ice in the patients randomized to spinal anesthesia with either 11.25 or $15 \mathrm{mg}$ of hyperbaric bupivacaine for Cesarean delivery and Cesarean delivery hypotension when intrathecal bupivacaine, fentanyl, and morphine are utilized. We found that this median seated time is dependent on bupivacaine dosage. A previous study determined the seated time for patients receiving hyperbaric bupivacaine and morphine without fentanyl. ${ }^{8}$ The addition of fentanyl appears to result in median seated times that are similar for lower doses of bupivacaine, but about one minute longer in higher doses. This could represent a synergistic effecti.e., fentanyl may exert a greater modulating effect on the relationship between seated time and hypotension in the presence of larger amounts of bupivacaine. The reasons for this are unclear. It may be related to an interaction between the longer seated time required for the 15-mg group and the reduced baricity of the intrathecal medication. It may also be due to direct pharmacologic interaction between the bupivacaine and fentanyl in the spinal cord. Further work is required to investigate this phenomenon. Importantly, the requirement for supplemental analgesia was 11/49 (22\%) in the previous study of intrathecal bupivacaine without fentanyl and 1/30 (3\%) in this current study which included fentanyl.

There are limitations to our findings. We studied nonobese patients scheduled for elective surgery, and our results may not reflect the results in other patient populations. We
Fig. 4 The isotonic regression of pooled-adjacent-violators algorithm (PAVA) and observed probability of avoiding hypotension at each examined seated time in patients randomized to spinal anesthesia with either 11.25 or $15 \mathrm{mg}$ of hyperbaric bupivacaine for Cesarean delivery

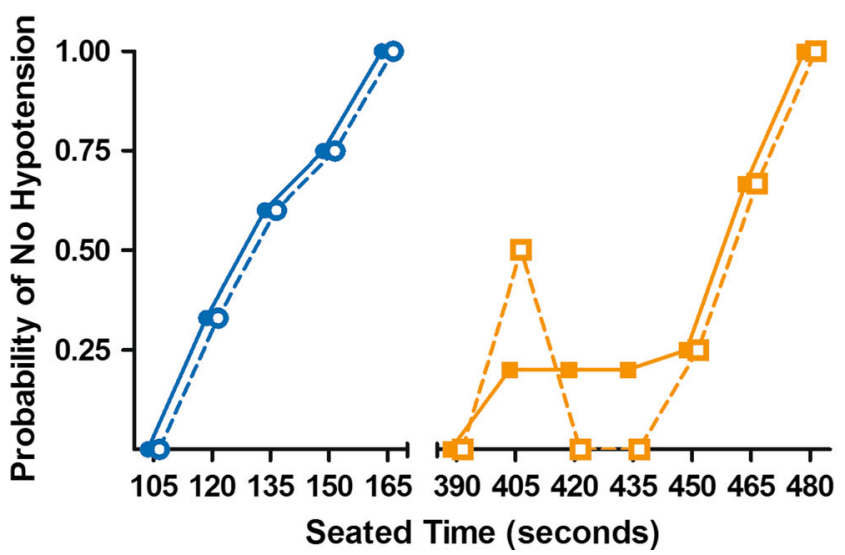

$-11.25 \mathrm{mg}$ PAVA

-o. 11.25 Observed

- 15 mg PAVA $15 \mathrm{mg}$ Observed 
utilized a combined spinal-epidural technique. Although we limited the amount of fluid injected into the epidural space, its presence could influence the spread of local anesthesia in the intrathecal space, ${ }^{16}$ and our results might not apply to patients receiving a single-shot spinal anesthetic. Simple updown techniques can provide reliable estimates of median effectiveness because the majority of patients are exposed to levels of treatment that are close to the median value. Nevertheless, this methodology provides much less reliable estimates for other levels of effectiveness. ${ }^{13}$ Therefore, the 95\% effective seated time should be interpreted with caution. Along these same lines, to ensure we had an adequate number of patients in the 15-mg group exposed to doses around the median value, we chose to continue enrolment after we reached six crossovers. We made this decision because the earliest crossover occurred farther away from where the later crossovers clustered. Ignoring the initial "run" up to where patients cluster is a recognized method of analysis in up-down methodology ${ }^{13}$ and can provide reliable estimates of median efficacy. ${ }^{17}$

We chose to determine a median effective point for seated time. Although a median effective point is useful for comparison of potency and an important point in doseresponse curves, a 90 or $95 \%$ effective point is more clinically useful. ${ }^{18}$ However, the methods required to determine effective points at the tails of a dose-response curve require significantly larger sample sizes than the simple up-down methods utilized for median effectiveness. ${ }^{13}$ We chose to determine a median effective seated time to allow comparison with the already determined median seated times for bupivacaine alone and to establish how intrathecal fentanyl would alter analgesic requirements while studying a minimal number of patients. Further work is required to determine the 90 or $95 \%$ effective seated time.

This was not a randomized comparison of extended seated time, and we cannot use these results to recommend this technique as clinically useful or as an alternative to the currently used techniques for prevention of hypotension. The clinical risks and benefits of extending seated time are not fully elucidated. Although it does appear to be associated with avoidance of hypotension, it also carries a risk of inadequate anesthesia. The level of this risk is currently unknown. For this reason, we utilized a combined spinal-epidural technique. In spite of this precaution, only one patient required supplemental analgesia after delivery. Also, one patient did not demonstrate a dermatomal blockade $15 \mathrm{~min}$ after what we thought was intrathecal injection. Failure of spinal anesthesia has many causes, ${ }^{19}$ including mistakenly identifying saline in the epidural space as cerebrospinal fluid. Nevertheless, the failure may also have been due to the extended seated time. In addition, the use of a combined spinal-epidural technique is more complicated than a single-shot spinal, and extending the seated time increases anesthesia procedure time. Although this technique may be useful in resource-poor settings or as an adjunct for prevention of hypotension, extended seated time requires further comparison with the many effective therapies currently utilized for hypotension before its clinical role can be determined.

We have determined the time required to remain seated after intrathecal injection of hyperbaric bupivacaine, morphine, and fentanyl to avoid hypotension in $50 \%$ of patients undergoing Cesarean delivery. Knowledge of this time is required to guide any further clinical examination of this technique.

\section{Competing interests None declared.}

Editorial responsibility This submission was handled by Dr. Philip M. Jones, Associate Editor, Canadian Journal of Anesthesia.

Author contributions Albert Moore, Aly el-Bahrawy, Roupen Hatzakorzian, and William Lipishan helped design the study. Albert Moore, Eva el-Mouallem, Aly el-Bahrawy, Mahmoud Moustafa, Simone Derzi, and Ian Kaufman helped perform the study. Albert Moore, Eva el-Mouallem, and Aly el-Bahrawy helped analyze the data. Albert Moore, Eva el-Mouallem, Aly el-Bahrawy, Ian Kaufman, Roupen Hatzakorzian, and William Lipishan helped write the manuscript.

Funding Departmental funds.

\section{References}

1. Hartmann B, Junger A, Klasen J, et al. The incidence and risk factors for hypotension after spinal anesthesia induction: an analysis with automated data collection. Anesth Analg 2002; 94: 1521-9.

2. Arzola $C$, Wieczorek $P$. Efficacy of low-dose bupivacaine in spinal anaesthesia for caesarean delivery: systematic review and meta-analysis. Br J Anaesth 2011; 107: 308-18.

3. Bamber JH, Dresner M. Aortocaval compression in pregnancy: the effect of changing the degree and direction of lateral tilt on maternal cardiac output. Anesth Analg 2003; 97: 256-8.

4. Loubert C. Fluid and vasopressor management for cesarean delivery under spinal anesthesia: continuing professional development. Can J Anesth 2012; 59: 604-19.

5. Allen TK, George RB, White WD, Muir HA, Habib AS. A doubleblind, placebo-controlled trial of four fixed rate infusion regimens of phenylephrine for hemodynamic support during spinal anesthesia for cesarean delivery. Anesth Analg 2010; 111: 1221-9.

6. Kohler F, Sorensen JF, Helbo-Hansen HS. Effect of delayed supine positioning after induction of spinal anaesthesia for caesarean section. Acta Anaesthesiol Scand 2002; 46: 441-6.

7. El-Hakeem EE, Kaki AM, Almazrooa AA, Al-Mansouri NM, Alhashemi JA. Effects of sitting up for five minutes versus immediately lying down after spinal anesthesia for cesarean delivery on fluid and ephedrine requirement; a randomized trial. Can J Anesth 2011; 58: 1083-9.

8. Moore A, Bourrassa-Blanchette S, El Mouallem E, et al. The median effective seated time for hypotension induced by spinal 
anesthesia at cesarean delivery with two doses of hyperbaric bupivacaine: a randomized up-down sequential allocation study. Can J Anesth 2014; 61: 916-21.

9. Dahl JB, Jeppesen IS, Jorgensen H, Wetterslev J, Moiniche S. Intraoperative and postoperative analgesic efficacy and adverse effects of intrathecal opioids in patients undergoing cesarean section with spinal anesthesia: a qualitative and quantitative systematic review of randomized controlled trials. Anesthesiology 1999; 91: 1919-27.

10. Unlugenc $H$, Ozalevli $M$, Gunduz $M$, et al. Comparison of intrathecal magnesium, fentanyl, or placebo combined with bupivacaine $0.5 \%$ for parturients undergoing elective cesarean delivery. Acta Anaesthesiol Scand 2009; 53: 346-53.

11. Shende D, Cooper GM, Bowden MI. The influence of intrathecal fentanyl on the characteristics of subarachnoid block for caesarean section. Anaesthesia 1998; 53: 706-10.

12. Paul M, Fisher DM. Are estimates of MAC reliable? Anesthesiology 2001; 95: 1362-70.

13. Pace NL, Stylianou MP. Advances in and limitations of up-anddown methodology: a precis of clinical use, study design, and dose estimation in anesthesia research. Anesthesiology 2007; 107: 144-52.

14. Stylianou M, Flournoy $N$. Dose finding using the biased coin upand-down design and isotonic regression. Biometrics 2002; 58: 171-7.

15. Stylianou M, Proschan M, Flournoy $N$. Estimating the probability of toxicity at the target dose following an up-and-down design. Stat Med 2003; 22: 535-43.

16. Takiguchi T, Okano T, Egawa H, Okubo Y, Saito K, Kitajima T. The effect of epidural saline injection on analgesic level during combined spinal and epidural anesthesia assessed clinically and myelographically. Anesth Analg 1997; 85: 1097-100.

17. Davis M. Comparison of sequential bioassays in small samples. J R Stat Soc Series B Stat Methodol 1971; 33: 78-87.

18. Fisher D. What if half of your patients moved (or remembered or did something else bad) at incision? Anesthesiology 2007; 107: 1-2.

19. Fettes PD, Jansson JR, Wildsmith JA. Failed spinal anaesthesia: mechanisms, management, and prevention. Br J Anaesth 2009; 102: 739-48. 